\title{
The data acquisition system of the NA62 experiment at CERN
}

\author{
Marco Boretto $^{1 *}$, on behalf of the NA62 Collaboration \\ ${ }^{1}$ Dipartimento di Fisica Sperimentale dell'Università e Sezione dell'INFN di Torino, I-10125 Torino, \\ Italy
}

\begin{abstract}
The aim of the NA62 experiment is to study the extreme rare kaon decay $K^{+} \rightarrow \pi^{+} v \bar{v}$ and to measure its branching ratio with a $10 \%$ accuracy. In order to do so, a very high intensity beam from the CERN SPS is used to produce charged kaons whose decay products are detected by many detectors installed along a $60 \mathrm{~m}$ decay region. The NA62 Data Acquisition system (DAQ) exploits a multi-level trigger system; following a Level0 (L0) trigger decision, $1 \mathrm{MHz}$ data rate from about 60 sources is read by a PC-farm, the partial event is built and then passed through a series of Level1 (L1) algorithms to further reduce the trigger rate. Events passing this level are completed with the missing, larger, data sources ( 400 sources) at the rate of $100 \mathrm{KHz}$. The DAQ is built around a high performance ethernet network interconnecting the detectors to a farm of 30 servers. After an overall description of the system design and the main implementation choices that allowed to reach the required performance and functionality, this paper describes the overall behaviour of the DAQ in the 2017 data taking period. It then concludes with an outlook of possible improvements and upgrades that may be applied to the system in the future.
\end{abstract}

\section{Introduction}

The NA62 experimental apparatus [1], located at the CERN Super Proton Synchrotron (SPS), has the main goal to measure the branching ratio of the $K^{+} \rightarrow \pi^{+} v \bar{v}$ decay with $10 \%$ precision. This process is extremely rare, with an estimated branching ratio of $\sim 10^{-10}$ [2]. The search for this process requires a high intensity kaon beam to collect sizable signal event samples, about $10^{13}$ kaon decays have to be collected over the data taking period. For that reason the Trigger and the Data Acquisition (TDAQ) chain requires a careful design in order to guarantee a high acceptance for the signal events, while keeping a high rejection of the background.

\subsection{The NA62 kaon beam}

The kaon beam is produced by impinging the $400 \mathrm{GeV}$ primary proton beam from SPS on a beryllium target. After the collision a $75 \mathrm{GeV}$ beam composed of $\pi^{+}, K^{+}$and protons is selected. The delivery of the beam follows the SPS spill cycle, it can be imagined like a periodic waveform in which the amplitude alternates at a steady frequency between 0 and the maximum value. The cycle followed by the SPS delivers 4.8 seconds of beam, called burst.

\footnotetext{
*e-mail: marco.boretto@edu.unito.it
} 
Bursts can be spaced by $\sim 40 \mathrm{~s}$ or $\sim 9.6 \mathrm{~s}$ depending on the SPS cycle. This break is exploited by the DAQ system to recover the initial state and be ready for the next burst. During 2017 the beam was operating at $60 \%$ of the nominal intensity.

\section{The NA62 trigger and DAQ systems}

The main DAQ infrastructure (Figure 1) is composed of a cluster of 30 servers (PC-farm) and 3 servers (Mergers). The Mergers collect the output from the PC-farm and prepare it for transfer to the CERN permanent storage in the computer center.

Each PC-farm node has $2 \mathrm{CPU}$ sockets with eight double threaded cores $(2.7 \mathrm{GHz})$ and 64 GB of RAM. A PC-farm node is equipped with 2 Network Interface Cards (NIC), one interface is $10 \mathrm{GbE}$ and is used to receive data from the detectors, and the second is a $1 \mathrm{GbE}$ NIC used to send the selected data to the Mergers. Each Merger has 15 TB RAID 5 storage and 2 CPU sockets with eight double threaded cores $(2.9 \mathrm{GHz})$. The Mergers are connected to the network with a $10 \mathrm{GbE}$ link and their $44 \mathrm{~TB}$ total storage capacity is capable to store the raw data up to $\sim 2.5$ days. The operating system chosen for all the DAQ servers is the CentOS7 (CC7) distribution provided by CERN [3]. The DAQ reads the inputs generated by several detectors. Each detector is composed by a variable number of readout boards based on FPGAs. The amount of boards per detector is given in table 1 and table 2. NA62 relies on a multi-stage trigger system composed by the L0 and the High Level Triggers (HLT). The HLT is divided in two levels (L1 and L2).

During the design process of NA62 the maximum L0 and L1 trigger rate has been estimated to be $\sim 1 \mathrm{MHz}$ and $\sim 100 \mathrm{KHz}$ respectively. A further reduction of a factor 10 should be achieved by the L2 trigger in order to have final rate of events of the order of $10 \mathrm{KHz}$. With those values in mind the DAQ was estimated to produce at most $3 \mathrm{~GB}$ per second.

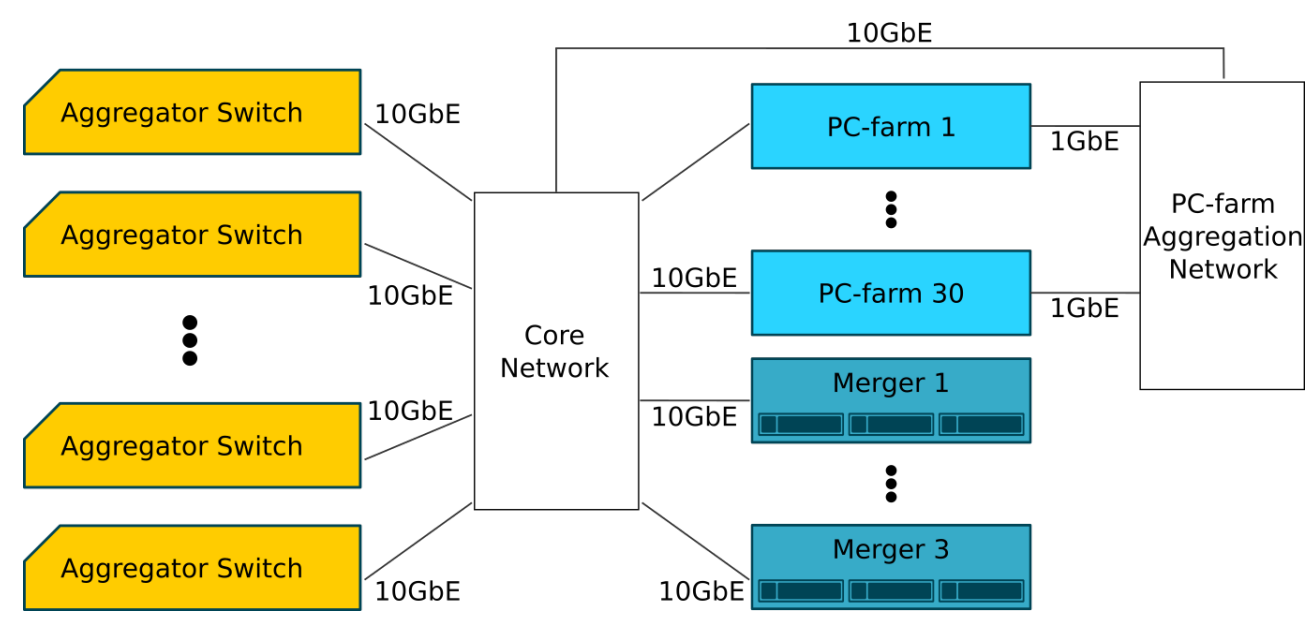

Figure 1. The scheme of the NA62 DAQ infrastructure. The Aggregator Switches distribute the data generated by the detectors during a burst to the entire PC-farm cluster. The output of the PC-farm is then conveyed by the PC-farm Aggregation Network and then routed to one of the 3 Mergers.

\subsection{The LO trigger}

The L0 is a hardware trigger, based on the input of a subset of detectors: CHOD, RICH, MUV and LKr. During a burst, each of those detectors contributes with data, called trigger 
Table 1. Number of DAQ boards per L0 detector.

\begin{tabular}{cc}
\hline Detector & \# DAQ boards \\
\hline CEDAR & 6 \\
\hline CHANTI & 2 \\
\hline LAV & 12 \\
\hline STRAW & 32 \\
\hline CHOD & 1 \\
\hline RICH & 5 \\
\hline IRC & 1 \\
\hline MUV3 & 1 \\
\hline HASC & 1 \\
\hline TOT & 61 \\
\hline
\end{tabular}

Table 2. Number of DAQ boards per L1 detector.

\begin{tabular}{cc}
\hline Detector & \# DAQ boards \\
\hline GTK & 6 \\
\hline LKr & 432 \\
\hline MUV1 & 6 \\
\hline MUV2 & 4 \\
\hline TOT & 448 \\
\hline
\end{tabular}

primitives, that allow $\mathrm{L} 0$ to form a decision. The primitives contain the detected activity characteristics and the time of occurrence. Primitives are sent via UDP packets to the L0 Trigger Processor (L0TP) [4], which can process data coming from a maximum of seven different sources. Up to $10 \mathrm{MHz}$ of primitives per detector can be handled by the system. The L0 trigger is configurable: in standard data taking conditions and beam intensity it generates about 2.3 M triggers per burst.

After a positive response from L0, data are read out from the 61 DAQ boards and sent to the PC-farm using the UDP protocol. Data from multiple events are stored in a UDP packet called MEP (Multi Event Packet), with a programmable packing factor. The NA62 readout packs together up to 8 events before sending a UDP packet to the PC-farm.

The UDP protocol has been chosen over the TCP protocol due to the simplicity of implementation in the front-end FPGAs. UDP (contrary to TCP) does not implement a flow control and just needs a source and destination port for the packets, without any acknowledgement or handshake: this eliminates all issues of communication establishment, at the cost of having to deal with data loss in case of congestion. To distribute the MEPs generated by each detector a round-robin scheduling on all the PC-farm nodes is performed.

\subsection{The network}

The components of the NA62 network can be grouped in 3 units: Aggregator Switches, Core Network and PC-farm Aggregation Network.

The Aggregator Switches are the first element of the network chain, they collect the output sent by the detectors on the $1 \mathrm{GbE}$ link and route it to the Core Network through $10 \mathrm{GbE}$ optical links. The number of Aggregator Switches per detector depends on the number of sources to read.

The main task of the Core Network is to route the traffic from the detectors to the PC-farm cluster. Internally the Core Network is composed by five routers: four $10-40 \mathrm{GbE}$ routers and one $40 \mathrm{GbE}$ router (Figure 2). The routers are built and optimized to accommodate enterprise network needs, which have continuous and constant traffic arriving randomly from each link. The NA62 case is exactly the opposite. The majority of the produced traffic is unidirectional, as it comes from the detectors and is also pulsed following the SPS spill period. For that reason the PC-farm and detector connections are distributed on different $10-40 \mathrm{GbE}$ routers. Furthermore with that configuration some traffic can already be routed locally, the rest have 
to be sent to the $40 \mathrm{GbE}$ router and routed to the proper destination. The Core Network can host up to $19210 \mathrm{GbE}$ links.

The PC-farm Aggregation Network is composed of a 1-10 GbE switch that collects the

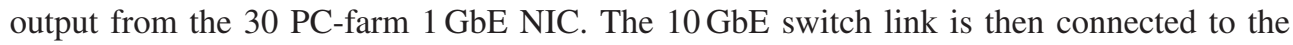
Core Network.

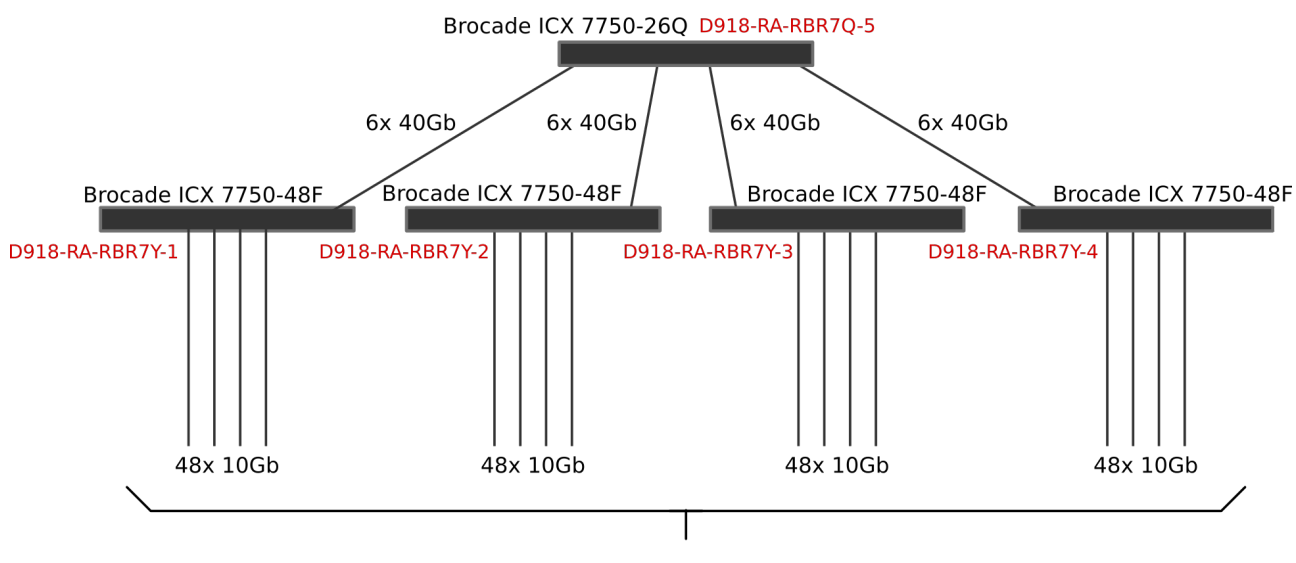

Links to PC-farm cluster, detectors and other NA62 servers

Figure 2. The scheme of the NA62 Core Network. The PC-farm and detectors are connected to the four Brocade ICX 7750-48F (10-40 GbE routers) which in turn are connected to the Brocade ICX 7750-26Q router (40 GbE router) [5].

\subsection{PC-farm cluster}

The NA62 data acquisition software is deployed on a cluster of 30 servers and is responsible of the following functions:

- build the L1 event;

- execute the HLT algorithms;

- send the request to complete the L2 event;

- build the L2 event;

- serialize the complete event.

\subsubsection{PC-farm Software}

The PC-farm software [6], also called DAQ software, is multi-threaded and written in C++ 11 [7]. The DAQ software, thanks to the round-robin scheduling, has to handle $1 / 30$ of the MEPs generated during the burst. During the PC-farm start-up a set of initialized events called Event Pool, capable to accommodate up to $200 \mathrm{~K}$ events, is preallocated. The PC-farm cluster should be able to collect all the $\sim 2.3 \mathrm{M}$ event triggered by the L0. This corresponds to $\sim 120 \mathrm{~K}$ MEPs per second per node.

To achieve the highest efficiency in collecting the UDP packets the DAQ software exploits PF_RING [8]: a library able to access the received packets directly from the NIC. To use this feature, the kernel network driver is replaced by the PF_RING one. PF_RING allows to scatter the incoming packets on many buffers in order to deliver them to different consumers 
on the application side. A pool composed by 6 threads is continuously collecting the packets from the $10 \mathrm{GbE}$ NIC on the 6 PF_RING buffers. Using the PF_RING non standard network stack means that many operations, usually handled by the kernel, need to be managed on the application side. For that reason the PC-farm software is also taking care of responding to ARP requests and to reassemble packets subjected to IP fragmentation. Furthermore the PC-farm software equipped with PF_RING has an exclusive usage of the $10 \mathrm{GbE}$ NIC. Any other service that exploit the network connectivity must use the $1 \mathrm{GbE}$ NIC.

Packets are then aggregated and delivered to a task manager which, after some checksum controls, assigns each fragment carried by the MEP to the corresponding event in the Event Pool. The Event Pool stores the pointers of the fragments arrived from the network that compose a physics event. This process is also called event building. There are two kinds of event building:

- The L1 event building produces the so-called L1 Event composed by the L0 detectors.

- The L2 event building produces the so-called L2 Event that adds the L1 fragments to the L1 Event.

The L1 Event for a specific event is considered complete once all the sources composing the L0 detectors have been received by the DAQ software. At this point the L1 trigger algorithms [9] can be run and the trigger decision evaluated. Each algorithm exploits information from individual detectors. The following L1 trigger algorithms were deployed in 2017:

- The KTAG L1 trigger uses the KTAG sector-multiplicity to positively identify a kaon and reject non-related accidental L0 triggers.

- The LAV L1 trigger uses a hit-multiplicity cut in eleven of the twelve LAV stations. It aims to reduce the background by identifying photons emitted at large angles. A cut requiring no more than two hits was applied to the events satisfying the L0 trigger.

- The STRAW L1 trigger reconstructs particle tracks by performing a two-dimensional Hough transform and a crude momentum evaluation. The number of tracks and the momentum are used to evaluate the trigger decision according to the physics mask triggered by the LO.

The typical amount of events passing the $\mathrm{L} 1$ algorithms is $\sim 250 \mathrm{~K}$ per burst. If the verdict from the L1 trigger algorithms is positive, the missing part of the event has to be requested from all the L1 sources. The event IDs to be requested are packed together in a Multiple Request Packet (MRP) and sent via a UDP packet through the $10 \mathrm{GbE}$ interface. It is important to mention that this is the only traffic directed from the PC-farm to the detectors. Data are read out from 448 DAQ boards of the L1 detectors and sent to the PC-farm through the Aggregation Switches and the Core Network. The L1 DAQ boards are not packing multiple events together, each event fragment is shipped alone in the UDP packet. It is estimated that $\sim 23 \mathrm{M}$ UDP packets per second are generated to complete all the L2 Events. To collect UDP packets generated from L0 and L1 detectors each PC-farm node has to handle a packet rate of about $\sim 900 \mathrm{KHz}$.

The acquisition process of the L1 sources in the PC-farm software is exactly the same as described for building the L1 Event. With the complete event it is possible to execute additional algorithms to further reduce the PC-farm output. The software is set up to host them, although no algorithms were applied. Trigger algorithms combining information from several detectors are foreseen to be deployed in the future.

Once the L2 Event is completed it is serialized and sent to one server of the Merger cluster across a ZMQ [10] socket. The mean size of the L2 Event is about $\sim 14 \mathrm{~KB}$. The event in the Event Pool is reset in order to be re-used in the next burst. L0 UDP packets allocated in 
memory cannot be freed right away, other event fragments carried by the MEP may be still needed to complete other events. The PC-farm output is sent through the $1 \mathrm{GbE}$ NIC with TCP packets to the PC-farm Aggregation Network that routes the packets back to the Core Network. The choice to use the $1 \mathrm{GbE}$ port rather than the $10 \mathrm{GbE}$ has been driven by the fact that it is extremely complex to implement the TCP protocol over PF_RING. Six seconds after the end of the burst the PC-farm software performs a cleanup of the Event Pool, incomplete events are deleted. After this operation the PC-farm cluster is ready to receive the subsequent burst.

\subsection{The merger cluster}

Fully reconstructed events collected during a burst are sent from the PC-farm nodes to one Merger. The PC-farm nodes execute a round-robin per burst on the 3 Mergers. The NA62 Merger software [11] is deployed on 3 servers and is responsible for the following functions:

- collect the serialized events from the 30 PC-farm nodes;

- collect additional metrics from the NA62 detectors;

- write the events and the metrics to a file.

\subsubsection{The merger software}

The Merger software is multi-threaded and written in $\mathrm{C}++11$. It has been designed to handle, in case of necessity, multiple bursts concurrently. For that reason the Mergers don't need to reset and be synchronized to the burst periodicity.

The events sent by the entire PC-farm cluster are polled from a $10 \mathrm{GbE}$ interface and organized in memory according to their burst number. A burst is considered complete if all event, belonging to that burst, are received in a $20 \mathrm{~s}$ period. At the same time some extra information from the experimental apparatus are fetched. The collection of the events on a single Merger allows to order all the events generated during a burst by event ID. The ordered events and the extra information received are written to file. Up to 4 bursts can be written simultaneously. The typical size of a raw file is $\sim 3.7$ GB per burst.

\subsubsection{The CDR system}

The task of the CDR system is to transfer the raw data from the DAQ to the CERN Advanced STORage manager [12] (CASTOR). It exploits the File Transfer Service [13] (FTS), the same technology used to move the data on the GRID. The CDR system is composed of:

- a book-keeping system;

- the globus-gridftp-server [14];

- and the CDR software.

The book-keeping system is composed of a MariaDB [15] database and its replica, those two elements are hosted on two different Merger hosts. In case of failure the replica allows to have the most up-to-date backup of the main database. The information of each file generated by the Merger software are stored into the database along with the transfer status (e.g. transferred, transferring..).

The CDR software is a single threaded software written in Python. It is deployed on all the Mergers with the following main functions:

- continuously seek new files generated by the Merger software; 
- update the file status on the database;

- schedule the file transfers.

The transmission on the network is handled by the globus-gridftp-server which is an additional service able to communicate and transfer data on the GRID.

All the files registered in the database must be transferred correctly on CASTOR. A raw file transfer is considered successful if the checksum of source and the destination match, on average a file is correctly transferred in $\sim 1.5$ minutes. In case of unsuccessful transfer the FTS service is configured to retry automatically the transfer 3 times. If the transfer is still unsuccessful after those 3 attempts, the CDR will take care of resubmitting it after 6 hours. For on-line monitoring purposes successfully transferred files are still kept on the Merger hosts for 24 hours. In case of interruption by the CASTOR service those files can be deleted in order to exploit all the storage capacity until the proper storage functioning is re-established.

\section{Conclusions}

This paper describes the NA62 DAQ system commissioned in the 2017 data-taking and the typical values at each trigger stage.

The Core Network has been successfully commissioned and has enhanced the network connectivity, upgrading the Mergers links from $1 \mathrm{GbE}$ to $10 \mathrm{GbE}$. Furthermore many spare ports are now available in case of failure. The PC-farm cluster equipped with PF_RING has demonstrated to be capable to sustain the packets rate from the experimental apparatus. During the PC-farm software initial development process, PF_RING was the only solution available to achieve high network throughput. In a possible upgrade of the PC-farm software, it would be interesting to explore a standard network solution in order to exploit the $10 \mathrm{GbE}$ NIC for the output traffic. This will allow the removal of the PC-farm Aggregation Network from the DAQ chain and also to have an easier and more maintainable code.

In 2017 the L1 data reduction achieved is $\sim 9$, however the L1 rate includes also the control trigger stream, which is not reduced. The L1 reduction factor taking in account just the physics trigger streams is $\sim 14$. With the described DAQ configuration the average data rate on disk, including the physics and control streams, was $\sim 0.8 \mathrm{~GB} / \mathrm{s}$. Due to the small event size and thanks to a high reduction factor at L1 it was possible to run the system even without the reduction from the L2 trigger. Possible upgrades of the system include the deployment of L2 trigger algorithms to further reduce the amount of events written on disk.

In order further to increase the rate from the experimental apparatus the UDP packet rate must be contained. This will be possible if the L1 detectors will group together multiple events in a single UDP packets, like the L0 detectors.

The CDR software described has replaced the previous legacy one. It has successfully transferred $\sim 730 \mathrm{~K}$ files to CASTOR, $90 \%$ of them in less than a minute. This is the first instance where the FTS technology is used to transfer data produced from a high energy physics experiment directly on the GRID.

\section{Acknowledgements}

We would like to thank Luca Deri and the NTOP developers for providing the PF_RING software and the precious support. We also want to thank CERN IT-CS team for their help in setting up the Core Network infrastructure. We would like to show our gratitude to the CERN IT-ST team for their help in setting up the FTS system and the efforts in monitoring it during all the data taking. 


\section{References}

[1] The NA62 Collaboration, Journal of Instrumentation 12, P05025 (2017)

[2] A.J. Buras, D. Buttazzo, J. Girrbach-Noe, R. Knegjens (2015)

[3] CERN CC7 http://linux.web.cern.ch/linux/centos7

[4] D. Soldi, S. Chiozzi, Journal of Instrumentation 13, P05004 (2018)

[5] Brocade Data sheet http://www.dataswitchworks.com/datasheets/brocade-icx-7750-ds. pdf

[6] NA62 PC-farm software https://github.com/NA62/na62-farm

[7] ISO/IEC. (2011). ISO International Standard ISO/IEC 14882:2011 - Programming Language C++. Geneva, Switzerland. http://www.open-std.org/jtc1/sc22/wg21/docs/ papers/2012/n3337.pdf

[8] PF_RING https://www.ntop.org/products/packet-capture/pf_ring

[9] NA62 trigger algorithms software https://github.com/NA62/na62-trigger-algorithms

[10] ZMQ http://zeromq.org

[11] NA62 Merger software https:/github.com/NA62/na62-farm-merger

[12] CASTOR CERN Advanced STORage manager http://castor.web.cern.ch

[13] FTS File Transfer Service https://fts.web.cern.ch

[14] Globus Grid FTP server https://www.globus.org

[15] MariaDB https://mariadb.org 